\title{
Towards an Effective Action for D-Branes
}

\author{
A. Keurentjes ${ }^{1}$, P. Koerber ${ }^{2}$, S. Nevens ${ }^{1}$, A. Sevrin ${ }^{*}$, A. Wijns ${ }^{1}$ \\ 1 Theoretische Natuurkunde, Vrije Universiteit Brussel and The International Solvay \\ Institutes, \\ Pleinlaan 2, B-1050 Brussels, Belgium \\ ${ }^{2}$ University of British Columbia, Department of Physics and Astronomy, \\ 6224 Agricultural Rd., Vancouver, B.C., V6T 1Z1, Canada
}

\begin{abstract}
We introduce and review several indirect methods to calculate the effective action for a single D-brane or a set of coinciding D-branes.
\end{abstract}

\section{Introduction}

The knowledge of the effective action for one or more D-branes is one of the few tools available to study D-brane dynamics. The effective world volume action for $n$ coinciding Dp-branes is, in leading order in $\alpha^{\prime}$, given by the $d=9+1, N=1$ supersymmetric $U(n)$ Yang-Mills action dimensionally reduced to $p+1$ dimensions [1]. For a single D-brane, $n=1$, the effective action is known to all orders in $\alpha^{\prime}$ in the limit of constant (or slowly varying) background fields: it is the $d=9+1, N=1$ supersymmetric Born-Infeld action, dimensionally reduced to $p+1$ dimensions, 2] -9 . Both bosonic and fermionic terms as well as the couplings to the bulk background fields are known. Derivative corrections were studied in [10] (using the partition function method), in 11] (using boundary conformal field theory) and 12 (using the Seiberg-Witten map). Modulo field redefinitions, it was shown that there are no two derivative corrections and a proposal for the four derivative corrections was made through all orders in $\alpha^{\prime}$.

For $n>1$, the situation is more involved. Requiring the background fields to be constant brings one, because of $D_{a} F_{b c}=0 \Rightarrow\left[F_{a b}, F_{c d}\right]=0$, back to the abelian situation. So here, one has to deal with derivative corrections right from the start. Only partial results are known. Indeed, there are no $\mathcal{O}\left(\alpha^{\prime}\right)$ corrections and the $\mathcal{O}\left(\alpha^{\prime 2}\right)$ corrections were calculated from open superstring amplitudes in [13. Requiring that certain BPS configurations solve the equations of motion allowed one to calculate both the $\alpha^{\prime 3}$ [14, and the $\alpha^{\prime 4}$ [15], corrections (see also the summarizing equations in [16]).

As a direct calculation starting from open string scattering amplitudes is technically very involved, indirect methods are called for. In this paper we will review some of those and outline some of the future strategies.

\footnotetext{
${ }^{*}$ corresponding author E-mail: Alexandre.Sevrin@vub.ac.be
} 


\section{Linear Constraints on Magnetic Fields}

One of the most powerful methods to construct the effective action for D-branes rests on the requirement that certain configurations which generalize $d=4$ instantons solve the equations of motion. In this section we review these configurations in the $\alpha^{\prime} \rightarrow 0$ limit.

The Yang-Mills equations of motion ${ }^{1}$,

$$
D_{b} F_{b a}=0
$$

are solved by virtue of the Bianchi identities if one imposes,

$$
F_{a b} \propto \varepsilon_{a b c d} F_{c d}
$$

where $\varepsilon_{a b c d}$ is completely anti-symmetric in its indices. If one insists on preserving Lorentz covariancy, one is limited to $d=4$ and one deals with instantons. In [17] the maximal subgroups of $S O(2 \mathrm{~m})$ for which $\varepsilon$ transforms as a scalar were catalogued. Two generic cases emerged: $U(m) \subset S O(2 m)$ and $S O_{+}(7) \subset S O(8)$. The latter is one of the $S O(7)$ subgroups of $S O(8)$ for which the vector and one of the spinor representations decompose as $8=8$, while the other spinor representation decomposes as $8=1 \oplus 7$. This result is clarified if one passes to complex coordinates. The equations of motion become,

$$
\begin{aligned}
0 & =D_{\beta} F_{\bar{\beta} \alpha}+D_{\bar{\beta}} F_{\beta \alpha} \\
& =D_{\alpha} F_{\bar{\beta} \beta}+2 D_{\bar{\beta}} F_{\beta \alpha},
\end{aligned}
$$

where we used the Bianchi identities. The equations of motions are solved provided one imposes,

$$
F_{\alpha \beta}=F_{\bar{\alpha} \bar{\beta}}=0, \quad F_{\alpha \bar{\alpha}}=0,
$$

where in the last equation a summation over $\alpha$ is understood. This corresponds to the case $S U(m) \subset S O(2 m)$. The first two equations are recognized as holomorphicity conditions while the last is the stability condition; eq. (41) defines a stable holomorphic bundle. Passing back to real coordinates, one verifies that for $m=2$, these are equivalent to the instanton equations. The exceptional case $S O_{+}(7) \subset S O(8)$ is also easily dealt with. Indeed taking $m=4$ and modifying eq. (4) to,

$$
F_{\alpha \beta}=\frac{1}{2} \varepsilon_{\alpha \beta \gamma \delta} F_{\bar{\gamma} \bar{\delta}}, \quad F_{\alpha \bar{\alpha}}=0,
$$

one again solves the equations of motion. These equations are known as the octonionic instanton equations which generalize the ordinary - also known as quaternionic - instanton equations. Turning back to real coordinates, eq. (5) can be rewritten as,

$$
F_{8 a}=\frac{1}{2} f_{a b c} F_{b c}, \quad a, b, c \in\{1, \cdots, 7\},
$$

with $f_{a b c}$ the (completely anti-symmetric) octonionic structure constants,

$$
f_{127}=f_{163}=f_{154}=f_{253}=f_{246}=f_{347}=f_{567}=1 .
$$

Written in this way, it is clear that this is the $d=8$ generalization of the well known $d=4$ (quaternionic) instantons,

$$
F_{4 a}=\frac{1}{2} \varepsilon_{a b c} F_{b c}, \quad a, b, c \in\{1,2,3\} .
$$

\footnotetext{
${ }^{1}$ We work in flat Euclidean space of dimension $d=2 m$. Unless stated otherwise, we sum over repeated indices regardless their position. In the next sections we will often put $2 \pi \alpha^{\prime}=1$.
} 
For $m \leq 4$, the solutions discussed above are BPS configurations of D-branes. For generic values of the magnetic fields satisfying eq. (44), one has 8, 4, 2 unbroken supersymmetries resp. for $m=2, m=3, m=4$ resp. The octonionic configuration, eq. (5) leaves a single supersymmetry unbroken. Explicit solutions to these equations can be interpreted as certain stable arrangements of D-branes. An exception on this are solutions of the octonionic type which generically have no concrete realization in terms of D-branes. However, even there some progress is being made. Indeed, starting e.g. from eq. (8) in the abelian limit and dimensionally reducing it to $d=3$, one obtains the Dirac monopole which can be viewed as a single D3-brane with a stack of D1-branes perpendicular to it. The number of D1-branes corresponds to the monopole number. This is the so-called BIon configuration [18], 19]. Similarly, eq. (51) in the abelian limit dimensionally reduced to $d=7$ can be reinterpreted as a single D7-brane with a configuration of D5-branes each having 4 directions longitudinal to the D7-brane. In addition several stacks of D3-branes and a stack of D1-branes can be added [20]. Their possible locations are determined by the octonionic structure constants.

Note that the BPS equations for $m>2$ do get $\alpha^{\prime}$ corrections. As these solutions have to solve the equations of motion of the effective action, it was suggested in [21] that they might provide a handle on the $\alpha^{\prime}$ corrections in the effective action. In the next section we will see that this is indeed the case.

\section{The Effective Action from Stable Holomorphic bundles}

A natural question which arises is whether we can deform the Yang-Mills action in such a way that stable holomorphic bundles - or some deformation of it - remain solutions to the equations of motion. As an example, we look at the abelian case and assume the fieldstrengths to be constant. We then add terms polynomial in the field strength to the action ignoring derivative terms, arriving at the following most general action through order $\alpha^{\prime 2}$

$$
\mathcal{S}=\frac{1}{g_{Y M}^{2}} \int\left(\frac{1}{4} \operatorname{tr} F^{2}+\lambda_{1} \operatorname{tr} F^{4}+\lambda_{2}\left(\operatorname{tr} F^{2}\right)^{2}+\mathcal{O}\left(\alpha^{\prime 4}\right)\right),
$$

with $\lambda_{1}$ and $\lambda_{2}$ arbitrary real coefficients ${ }^{2}$. From this the equations of motion follow,

$$
\begin{aligned}
0= & \partial_{\bar{\beta}}\left(F_{\alpha \bar{\alpha}}+\frac{8 \lambda_{1}}{3} F_{\alpha \bar{\alpha}}^{3}\right)+\left(4 \lambda_{1}+16 \lambda_{2}\right) \partial_{\bar{\gamma}} F_{\alpha \bar{\alpha}}^{2} F_{\gamma \bar{\beta}}+ \\
& 16 \lambda_{2} F_{\gamma \bar{\gamma}}^{2} \partial_{\bar{\beta}} F_{\alpha \bar{\alpha}}+8 \lambda_{1} F_{\gamma \bar{\beta}}^{2} \partial_{\bar{\gamma}} F_{\alpha \bar{\alpha}}+\mathcal{O}\left(\alpha^{\prime 4}\right),
\end{aligned}
$$

where we passed to complex coordinates, used the Bianchi identities and implemented the holomorphicity conditions. The first term vanishes provided we deform the stability condition,

$$
F_{\alpha \bar{\alpha}}+\frac{8 \lambda_{1}}{3} F_{\alpha \bar{\alpha}}^{3}+\mathcal{O}\left(\alpha^{\prime 4}\right)=0
$$

while the second term vanishes if,

$$
\lambda_{2}=-\frac{1}{4} \lambda_{1}
$$

The stability condition in leading order takes care that the remainder vanishes as well, but because of eq. (11) they will make a contribution at order $\alpha^{\prime 4}$ thereby relating $\lambda_{1}$ to the arbitrary coefficients at that order.

\footnotetext{
${ }^{2} F_{a b}^{l}$ stands for $F_{a c_{1}} F_{c_{1} c_{2}} \cdots F_{c_{l-1} b}$ and $\operatorname{tr} F^{l} \equiv F_{a a}^{l}$.
} 
We see that at this point there are two undetermined coefficients left: an overall multiplicative constant $1 / g_{Y M}^{2}$ and $\lambda_{1}$. The latter can be absorbed by rescaling $F$, putting $\lambda_{1}=1 / 8$, its conventional value. Proceeding to higher orders, one finds that all coefficients get uniquely fixed, yielding the Born-Infeld action [22]!

This method can be extended to the non-abelian case as well. In this way the action was obtained through order $\alpha^{\prime 4}$ [14, [15. Modulo field redefinitions it is given by,

$$
\mathcal{L}=\frac{1}{g^{2}}\left(\mathcal{L}_{0}+\mathcal{L}_{2}+\mathcal{L}_{3}+\mathcal{L}_{4}\right)
$$

where the leading term is simply ${ }^{3}$

$$
\mathcal{L}_{0}=-\operatorname{Tr}\left\{\frac{1}{4} F^{2}\right\}
$$

Subsequently we have

$$
\mathcal{L}_{2}=\operatorname{STr}\left\{\frac{1}{8} F^{4}-\frac{1}{32} F^{2} F^{2}\right\},
$$

and

$$
\mathcal{L}_{3}=\frac{\zeta(3)}{2 \pi^{3}} \operatorname{Tr}\left\{\left[D_{3}, D_{2}\right] D_{4} F_{51} D_{5}\left[D_{4}, D_{3}\right] F_{12}\right\}
$$

The overall coefficient of this term remained undetermined ${ }^{4}$ when using the method of 22. It was fixed by comparing it to the partial result for this term in 23 obtained by a direct string theoretic calculation. Finally the fourth order term is completely determined by the method of [22] and it is given by,

$$
\mathcal{L}_{4}=\mathcal{L}_{4,0}+\mathcal{L}_{4,2}+\mathcal{L}_{4,4}
$$

with

$$
\begin{aligned}
\mathcal{L}_{4,0} & =-\operatorname{STr}\left(\frac{1}{12} F_{12} F_{23} F_{34} F_{45} F_{56} F_{61}-\frac{1}{32} F_{12} F_{23} F_{34} F_{41} F_{56} F_{65}+\frac{1}{384} F_{12} F_{21} F_{34} F_{43} F_{56} F_{65}\right) \\
\mathcal{L}_{4,2} & =-\frac{1}{48} \operatorname{STr}\left(-2 F_{12} D_{1} D_{6} D_{5} F_{23} D_{6} F_{34} F_{45}-F_{12} D_{5} D_{6} F_{23} D_{6} D_{1} F_{34} F_{45}\right. \\
& +2 F_{12}\left[D_{6}, D_{1}\right] D_{5} F_{23} F_{34} D_{4} F_{56}+3 D_{4} D_{5} F_{12} F_{23}\left[D_{6}, D_{1}\right] F_{34} F_{56} \\
& +2 D_{6}\left[D_{4}, D_{5}\right] F_{12} F_{23} D_{1} F_{34} F_{56}+2 D_{6} D_{5} F_{12}\left[D_{6}, D_{1}\right] F_{23} F_{34} F_{45} \\
& +2\left[D_{6}, D_{1}\right] D_{3} D_{4} F_{12} F_{23} F_{45} F_{56} \\
& \left.+\left[D_{6}, D_{4}\right] F_{12} F_{23}\left[D_{3}, D_{1}\right] F_{45} F_{56}\right) \\
& =-\frac{1}{1440} \operatorname{STr}\left(D_{6}\left[D_{4}, D_{2}\right] D_{5} D_{5}\left[D_{1}, D_{3}\right] D_{6} F_{12} F_{34}+4 D_{2} D_{6}\left[D_{4}, D_{1}\right]\left[D_{5},\left[D_{6}, D_{3}\right]\right] D_{5} F_{12} F_{34}\right. \\
& +2 D_{2}\left[D_{6}, D_{4}\right]\left[D_{6}, D_{1}\right] D_{5}\left[D_{5}, D_{3}\right] F_{12} F_{34}+6 D_{2}\left[D_{6}, D_{4}\right] D_{5}\left[D_{6}, D_{1}\right]\left[D_{5}, D_{3}\right] F_{12} F_{34} \\
& +4 D_{6} D_{5}\left[D_{6}, D_{4}\right]\left[D_{5}, D_{1}\right]\left[D_{4}, D_{3}\right] F_{12} F_{23}+4 D_{6} D_{5}\left[D_{4}, D_{2}\right]\left[D_{6}, D_{1}\right]\left[D_{5}, D_{3}\right] F_{12} F_{34} \\
& +4 D_{6}\left[D_{5}, D_{4}\right]\left[D_{3}, D_{2}\right]\left[D_{5},\left[D_{6}, D_{1}\right]\right] F_{12} F_{34} \\
& \left.+2\left[D_{6}, D_{1}\right]\left[D_{2}, D_{6}\right]\left[D_{5}, D_{4}\right]\left[D_{5}, D_{3}\right] F_{12} F_{34}\right) .
\end{aligned}
$$

\footnotetext{
${ }^{3}$ The $u(n)$ generators are taken to be hermitian. We always trace over the Lorentz indices and Tr and STr resp. stand for the group trace and the symmetrized group trace. When symmetrizing, we take $D^{l} F$ as a single unit.

${ }^{4}$ This is fortunate, as our method can only generate rational coefficients while $\zeta(3) / \pi^{3}$ is very probably an irrational number.
} 
It is clear that the result is quite complicated. In this form it does not particularly suggest any all order expression. The lesson which can be learned here is that it might be a good idea to return to the simpler abelian case and try to obtain control over the derivative corrections. Precisely this will be tackled in the next section.

\section{The Effective Action from $\beta$-Functions}

In this section we turn to an alternative way to recover the effective action. We consider an open string $\sigma$-model in a $U(1)$ background and require the model to be conformally invariant at the quantum level, i.e. we require the $\beta$-functions to vanish which is viewed as an equation of motion which subsequently must be integrated to the effective action. The $n$-loop contribution to the $\beta$-functions gives $2 n-2$-derivative corrections to the effective action. A great advantage of this approach is that the results are always all order in $\alpha^{\prime}$. At lowest order, this was already studied in [4], see also [24]. The calculations are greatly simplified when formulating the model in $N=2$ boundary superspace. In this setting the whole $U(1)$ structure is characterized by a single scalar potential $V$. The model at hand is a special case of the general setup developed in 25.

We introduce chiral fields, $Z^{\alpha}$, and anti-chiral fields, $Z^{\bar{\alpha}}, \alpha \in\{1, \cdots m\}$, satisfying the constraints,

$$
\bar{D} Z^{\alpha}=D Z^{\bar{\alpha}}=0
$$

In addition we need a set of fermionic constrained fields $\Psi^{\alpha}$ and $\Psi^{\bar{\alpha}}$, which satisfy,

$$
\bar{D} \Psi^{\alpha}=\partial_{\sigma} Z^{\alpha}, \quad D \Psi^{\bar{\alpha}}=\partial_{\sigma} Z^{\bar{\alpha}} .
$$

The action, $\mathcal{S}$, consists of a free bulk term, $\mathcal{S}_{0}$, and a boundary interaction term, $\mathcal{S}_{\text {int }}$,

$$
\mathcal{S}=\mathcal{S}_{0}+\mathcal{S}_{\text {int }},
$$

where,

$$
\begin{aligned}
\mathcal{S}_{0} & =\int d^{2} \sigma d^{2} \theta\left(g_{\alpha \bar{\beta}} D Z^{\alpha} \bar{D} Z^{\bar{\beta}}+g_{\alpha \bar{\beta}} \Psi^{\alpha} \Psi^{\bar{\beta}}\right), \\
\mathcal{S}_{i n t} & =-\int d \tau d^{2} \theta V(Z, \bar{Z}) .
\end{aligned}
$$

In these equations, we rescaled $Z$ ( $\Psi$ resp.) by a factor $\sqrt{2 \pi \alpha^{\prime}}$ such as to make it dimensionless (of dimension 1/2 resp.). We choose Neumann boundary conditions,

$$
\left.\Psi^{\alpha}\right|_{\text {boundary }}=\left.\Psi^{\bar{\alpha}}\right|_{\text {boundary }}=0 \text {. }
$$

The boundary term gives the coupling to a $U(1)$ background field. The magnetic fields, appropriately rescaled by a factor $2 \pi \alpha^{\prime}$, are obtained from the potential,

$$
\begin{aligned}
& F_{\alpha \bar{\beta}}=i V_{\alpha \bar{\beta}} \\
& F_{\alpha \beta}=F_{\bar{\alpha} \bar{\beta}}=0 .
\end{aligned}
$$

Calculating and resumming the UV divergent one loop contributions yields, using minimal subtraction, a counterterm,

$$
V_{\text {bare }}=V+i \frac{\ln \mu}{\pi} g^{\alpha \bar{\beta}}(\operatorname{arcth} F)_{\alpha \bar{\beta}},
$$


with $\mu$ the UV cut-off. Requiring the one-loop $\beta$-function to vanish yields,

$$
\mathcal{G}^{b c} \partial_{b} F_{c a}=0
$$

where $\mathcal{G}$ is defined by,

$$
\mathcal{G}^{a c}\left(g_{c b}-\left(F^{2}\right)_{c b}\right)=\delta_{b}^{a} .
$$

We immediately recognize eq. (26) as the equation of motion for the Born-Infeld action. Proceeding to the next order one finds that the $\ln \mu$ divergences cancel between the two-loop diagrams and the subdivergent diagram, leaving a $(\ln \mu)^{2}$ divergence. As a consequence, the $\beta$-function does not receive a two-loop contribution. Put differently: there are no two-derivative terms in the effective action, a result already derived in [10]. Presently the 3- and 4-loop contributions are being calculated and all order properties are being investigated [26].

\section{Conclusions}

It is clear that an all order expression for the effective action for D-branes will not be available in an immediate future. However, combining various indirect methods such as deformed stable holomorphic bundles and $\sigma$-model $\beta$-functions will probably lead to concrete results for at least the abelian case.

Acknowledgement We thank Klaus Behrndt, Marc Grisaru and Chris Hull for useful discussions. SN and AW are "FWO aspiranten". AK, SN, AS and AW are supported in part by the "FWO-Vlaanderen" through project G.0034.02, in part by the Belgian Federal Science Policy Office through the Interuniversity Attraction Pole P5/27 and in part by the European Commission FP6 RTN programme MRTN-CT-2004-005104. PK is supported by the Natural Sciences and Engineering Research Council of Canada.

\section{References}

[1] E. Witten, Bound states of strings and p-branes, Nucl. Phys. B460 (1996) 35, hep-th/9510135.

[2] E.S. Fradkin and A.A. Tseytlin, Nonlinear electrodynamics from quantized strings, Phys. Lett. B163 (1985) 123.

[3] A.A. Tseytlin, Vector field effective action in the open superstring theory, Nucl. Phys. B276 (1986) 391 and Nucl. Phys. B291 (1987) 876.

[4] A. Abouelsaood, C. Callan, C. Nappi and S. Yost, Open strings in background gauge fields, Nucl. Phys. B280 (1987) 599.

[5] R.G. Leigh, Dirac-Born-Infeld action from Dirichlet sigma model, Mod. Phys. Lett. A4 (1989) 2767.

[6] M. Cederwall, A. von Gussich, B. E. W. Nilsson and A. Westerberg, The Dirichlet super-three-brane in ten-dimensional type-IIB supergravity, Nucl. Phys. B490 (1997) 163, hep-th/9610148.

[7] M. Aganagic, C. Popescu and J. H. Schwarz, D-brane actions with local kappa symmetry, Phys. Lett. B393 (1997) 311, hep-th/9610249 and Gauge-invariant and gaugefixed D-brane actions, Nucl. Phys. B495 (1997) 99, hep-th/9612080. 
[8] M. Cederwall, A. von Gussich, B. E. W. Nilsson, P. Sundell and A. Westerberg, The Dirichlet super p-branes in ten-dimensional type IIA and IIB supergravity, Nucl. Phys. B490 (1997) 179, hep-th/9611159.

[9] E. Bergshoeff and P. K. Townsend, Super D-branes, Nucl. Phys. 490 (1997) 145, hep-th/9611173.

[10] O.D. Andreev and A.A. Tseytlin, Partition function representation for the open superstring effective action: cancellation of Möbius infinities and derivative corrections to Born-Infeld Lagrangian, Nucl. Phys. 311 (1988) 205.

[11] N. Wyllard, Derivative corrections to D-brane actions with constant background fields, Nucl. Phys. 598 (2001) 247, hep-th/0008125.

[12] L. Cornalba, The general structure of the non-abelian Born-Infeld action, Adv. Theor. Math. Phys. 4 (2002) 1259, hep-th/0006018.

[13] D. J. Gross and E. Witten, Superstring modifications of Einstein's equations, Nucl. Phys. B277 (1986) 1.

[14] P. Koerber and A. Sevrin, The non-abelian open superstring effective action through order $\alpha^{\prime 3}$, JHEP 0110 (2001) 003, hep-th/0108169

[15] P. Koerber and A. Sevrin, The non-abelian D-brane effective action through order $\alpha^{\prime 4}$, JHEP 0210 (2002) 046, hep-th/0208044

[16] A. Sevrin and A. Wijns, Higher order terms in the non-abelian D-brane effective action and magnetic background fields, JHEP 0308 (2003) 059, hep-th/0306260.

[17] E. Corrigan, C. Devchand, D. B. Fairlie and J. Nuyts, First Order Equations For Gauge Fields In Spaces Of Dimension Greater Than Four, Nucl. Phys. B214 (1983) 453.

[18] C. G. Callan and J. M. Maldacena, Brane dynamics from the Born-Infeld action, Nucl. Phys. B513 (1998) 198, hep-th/9708147.

[19] G. W. Gibbons, Born-Infeld particles and Dirichlet p-branes, Nucl. Phys. B514 (1998) 603, hep-th/9709027.

[20] A. Keurentjes, P. Koerber, A. Sevrin and A. Wijns, in preparation.

[21] F. Denef, A. Sevrin and J. Troost, Non-Abelian Born-Infeld versus string theory, Nucl. Phys. B581 (2000) 135, hep-th/0002180.

[22] L. De Fossé, P. Koerber and A. Sevrin, The uniqueness of the Abelian Born-Infeld action, Nucl. Phys. B603 (2001) 413, hep-th/0103015.

[23] A. Bilal Higher derivative corrections to the non-abelian Born-Infeld action, Nucl. Phys. B618 (2001) 21, hep-th/0106062.

[24] K. Behrndt Untersuchung der Weyl-Invarianz im verallgemeinerten $\sigma$-Modell für offene Strings, PhD-thesis, 1990, Humboldt University Berlin.

[25] P. Koerber, S. Nevens and A. Sevrin, Supersymmetric non-linear sigma-models with boundaries revisited, JHEP 11 (2003) 066, hep-th/0309229.

[26] S. Nevens, A. Sevrin and A. Wijns, in preparation. 